\title{
TENTATIVAS INOVADORAS NA PRÁtICA DE ENSINO E ASSISTÊNCIA NA ÁREA DE SAÚDE MENTAL - I
}

\section{THE CHANGING PRACTICE AND TEACHING IN PSYCHIATRIC NURSING}

\author{
Sônia Barros * \\ Ana Luisa Aranha e Silva ** \\ Ana Cristina Delgado Lopérgolo *** \\ Ana Maria Fernandes Pitta ****
}

BARROS, S. et al. Tentativas inovadoras na prática de ensino de assistência na área de saúde mental - I. Rev.Esc.Enf.USP, v.33, n.2, p. 192-9, jun. 1999 .

\section{RESUMO}

No atual contexto de transformações no campo da assistência à saúde mental no Brasil, a Universidade tem a responsabilidade de redirecionar a qualificação profissional da força de trabalho em saúde. Este artigo versa sobre a. experiência de um Programa de Integração Docente-Assistencial envolvendo a Secretaria de Estado de Saúde e a Universidade de São Paulo. Particulariza a experiência de estudantes de enfermagem cursando a disciplina de enfermagem psiquiátrica. A análise da avaliação cios alunos do programa teórico-prático desenvolvido na unidade, ancorado nos pressupostos da Reabilitação Psicossocial indica, ao mesmo tempo, que o modelo de atenção interfere diretamente na qualificação das práticas $e$, simultaneamente, desenvolve nos alunos atitudes $e$ conhecimentos que os qualificam para desempenhar de forma coerente suas funções.

UNITERMOS: Programa de integração docente-assistencial. Reabilitação psicossocial. Ensino de enfermagem psiquiátrica.

\begin{abstract}
In the context of recent changes that are taking place in the field of mental health care in Brazil, the University has the responsibility to redimensioning human, resources qualification of mental health workers: At the Mental Health Care and Educational Program. of the University of São Paulo and São Paulo Health Office (São Paulo/Brazil), an experience still in course, the nursing students attending to psychiatric nurse training evaluated that theoreticalpractical education, dealing with health care issues in the field of Psychosocial Rehabilitation purpose, at the same time that directly interferes in the qualification of practices, simultaneously develops the students attitudes and knowledge that qualify them to perform coherent practices in accordance with rehabilitation models of care.
\end{abstract}

UNITERMS: Teaching care integration program. Psychosocial rehabilitation. Teaching in psychiatric nursing.

\section{PROCESSO DE IMPLANTAÇÃO DO PROGRAMA DE INTEGRAÇÃO DOCENTE- ASSISTENCIAL EM SAÚDE MENTAL - PIDA/CAPS - SES/USP.}

Uma das diretrizes da atual política de saúde mental no país é estimular práticas de ensino, pesquisa e extensão que possibilitem aos futuros profissionais, novas atitudes em relação ao processo saúde-doença mental. A partir dessa diretriz, podemos afirmar que a mudança na qualidade de

\footnotetext{
Professor Doutor do Departamento de Enfermagem Materno-Infantil e Psiquiátrica da Escola de Enfermagem da USP. Email: sobarros@usp.br

** Doutoranda em Enfermagem. Professor assistente da disciplina de Enfermagem Psiquiátrica e em Saúde Mental da Faculdade de Enfermagem da Universidade de Mogi das Cruzes. Enfermeira o CAPS.

*** Mestranda no Núcleo de Subjetividade da Faculdade de Psicologia da Pontifícia Universidade Católica de São Paulo. Psicologa do CAPS.

**** Professor Doutor o Departamento de Medicina Preventiva da Faculdade de Medicina da Universidade de São Paulo.
} 
assistência à saúde passa, também, pelo redirecionamento de qualificação da força de trabalho em saúde e que as escolas têm responsabilidades na formação de atores comprometidos com projetos de transformação (BARROS, 1996).

Este entendimento é corroborado por CAVALCANTI (1993) quando afirma que "sem sujeitos em transformação, movidos por uma prática em transformação e ao mesmo tempo motores dessa prática, não há nenhuma possibilidade de transformações na assistência psiquiátrica”.

A enfermagem psiquiátrica é uma prática social vinculada $\mathrm{s}$ demais práticas em sáude $\mathrm{e}$, por isso, tem funcionado como uma caixa de ressonância das transformações pelas quais passa a assistência à saúde mental no país. Nesse processo de transformação, relacionado à prática e ao ensino, pretende-se que o objeto de assistência seja o sujeito, tomado na sua dimensão subjetiva e na dimensão interpessoal, potencializando sua capacidade de ser saudável.

Dessa forma, torna-se necessário um afinamento entre o instrumento (saber), o objeto de cuidado e o modelo assistencial. Para superar a dicotomia, apontada em diversos estudos realizados por enfermeiros, entre o saber reproduzido na Escola de Enfermagem e o fazer (ações práticas do enfermeiro) torna-se necessário que a "Escola coloque o seu saber na concretude do meio sem perder o seu caráter científico e especulativo" (REZENDE, 1986).

A consciência dessa contradição e a necessidade de apreender e construir novas concepções e novas práticas interventivas em saúde mental, determinou que docentes da disciplina Enfermagem Psiquiátrica tornassem partícipes de um Programa de Integração Docente-Assistencial (PIDA) em Saúde Mental no Centro de Atenção Psicossocial Professor Luiz da Rocha Cerqueira - o CAPS Itapeva, buscando superar o modelo tradicional de ensino e de assistência na enfermagem psiquiátrica.

A Escola de Enfermagem da Universidade de São Paulo, EEUSP, tem tradição em projetos de Integração Docente-Assistecial, IDA. A experiência mais recente, e que teve longa duração, foi desenvolvida pela disciplina de Enfermagem em Saúde Coletiva. Várias modalidades de integração entre ensino e serviço foram implementadas, envolvendo também a disciplina de Enfermagem Psiquiátrica e cujos pressupostos articuladores foram:

1.Estabelecimento de um processo de reflexão critica conjunta sobre a qualidade da assistência e ensino na área de saúde coletiva.;
2.0 processo de reflexão crítica deveria envolver efetivamente todos os integrantes, isto é, deveria ser um processo participativo de todos os componentes em todas as instâncias, portanto, o trabalho deveria ser regido pelo PRINCIPIO DA CONSTRUÇÃO COLETIVA.

3. Por envolver profissionais e ocupacionais de campo, docentes, discentes e representantes da comunidade, o trabalho seria regido pelo PRINCÍPIO DA RESPONSABILIDADE COMPARTILHADA, ou seja, ambas as instituições comprometidas com o desenvolvimento $e$ resultado do PIDA (UNIVERSIDADE, 1988).

Para assumir o atual Projeto da Saúde Mental, as docentes da disciplina de Enfermagem Psiquiátrica contaram com os conhecimentos acumulados e experiências adquiridas no PIDA Saúde Coletiva e assumiram os pressupostos acima mencionados, ainda que reconhecessem que estes estevam num estágio de reinterpretação pelos parceiros do PIDA Saúde Mental.

0 atual convênio foi firmado em 31 de outubro de 1996 e o locus do PIDA em Saúde Mental integra um Centro de Atenção Psicossocial e um Ambulatório de Saúde Mental da Secretaria de Estado da Saúde (SES); o Departamento de Medicina Preventiva da Faculdade de Medicina, o Curso de Terapia Ocupacional e a disciplina Enfermagem MaternoInfantil e Psiquiátrica da Escola de Enfermagem da Universidade de São Paulo.

O Centro de Atenção Psicossocial Prof. Luiz da Rocha Cerqueira (nome do psiquiatra semeador de reformas psiquiátricas pelo Brasil), conhecido como CAPS Itapeva - que delimita este complexo de assistência ampliado - é uma estrutura de atendimento substitutiva à hospitalocêntrica e tem por finalidade oferecer tratamento a pessoas acometidas por transtornos psíquicos (BARIÇÃO, 1994).

Neste serviço, "o modelo de tratamento desenvolvido diferencia-se do modelo psiquiátrico tradicional, porque os profissionais privilegiam e objetivam o olhar sobre o sujeito, compreendem a doença mental como um elemento limitador da expressão da subjetividade e das potencialidades humanas e buscam instrumentos de intervenção que ampliem seus repertórios terapêuticos com a finalidade de abrir fendas nesse limite, intervindo, assim, no processo saúde-doença" (SILVA, 1997).

Além disso, pretende-se que o Projeto privilegie a Reabilitação Psicossocial como campo de prática e conhecimento, que considere a capacidade dos sujeitos acometidos por transtornos psiquiátricos graves de construir uma possibilidade de contrato psicológico e social com o meio exterior. Nessa perspectiva, poderá formar profissionais 
capazes de operar um sistema de saúde, uma sociedade de cidadãos, que deverá assinar pesquisas onde estudos etiológicos, de planejamento, de gestão de avaliação, estejam permanentemente dirigidos às três vocações do projeto: assistência, ensino e pesquisa (PITTA et al, 1994).

No atual contexto, o organograma institucional está assim representado:

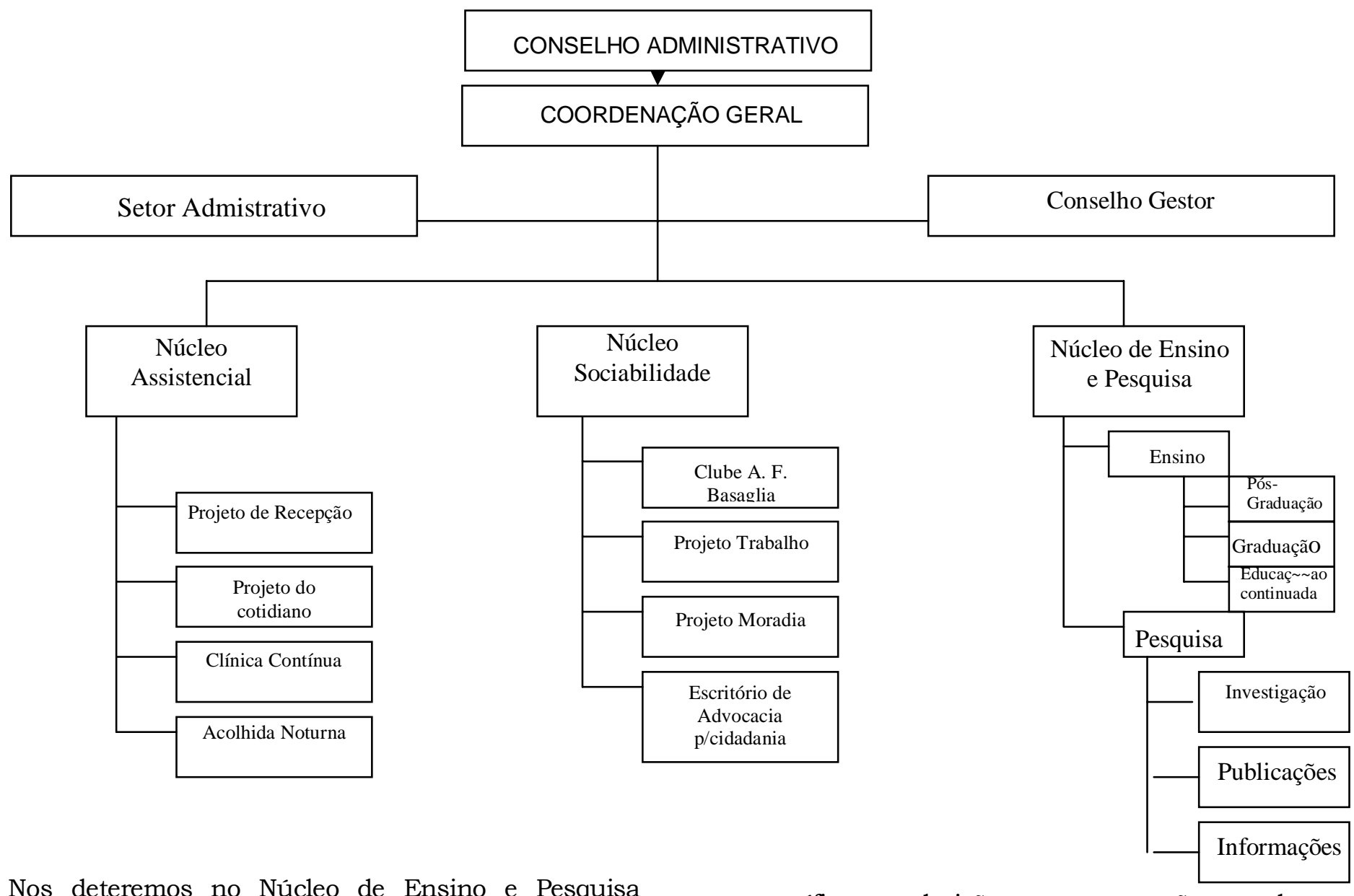

Nos deteremos no Núcleo de Ensino e Pesquisa (NEP). O CAPS foi inaugurado com vocação de institutição de ensino e, para tanto, contava com um Núcleo de Ensino e Pesquisa operacionalizado por técnicos do próprio serviço. Desde 1990 oferecia Curso de Aprimoramento Multiprofissional em Saúde Mental; viabilizava estágios de graduação de Psicologia da Pontificia Universidade Católica de São Paulo e de Terapia Ocupacional da Universidade de São Paulo; e investigações científicas de alunos de pós-graduação.

Com a vigência do PIDA, o NEP foi ampliado e a área do Ensino tornou-se um núcleo coordenado pela docente da disciplina de Enfermagem Psiquiátrica da Escola de Enfermagem; conta com uma psicóloga que coordena o aprimoramento; uma enfermeira que coordena os estágios de graduação e um psiquiatra que organiza a recepção de visitantes que vêm ao CAPS com objetivos diversos. O trabalho do grupo tem sido bastante fecundo, solidário e democrático. Em que pesem responsabilidades específicas, as decisões e propostas são tomadas pelo grupo que se reúne, no mínimo, uma hora e meia por semana.

Nos limites desse trabalho, nos interessa avaliar como foi o processo de aprendizagem de um grupo de alunos de enfermagem, que realizou o estágio (ensino teórico-prático) no CAPS quando já estávamos desenvolvendo o PIDA.

\section{AVALIAÇÃO DA EXPERIÊNCIA DE ALU- NOS DE GRADUAÇÃO DE ENFERMAGEM NO PIDA EM SAÚDE MENTAL}

A disciplina Enfermagem Psiquiátrica na Saúde do Adulto II, do curso de graduação em enfermagem da EEUSP é oferecida aos alunos a partir do $3^{\circ}$ semestre. 0 total de créditos corresponde a 150 horas de ensino, sendo 60 horas de auas teóricas e 90 horas de teórico-prática (estágio). 
Atualmente, para cumprir o estágio, o grupo de alunos é dividido em 05 sub-grupos que vai, cada um deles, para campos assistenciais diferentes por 06 ou 07 semanas, resultando cerca de 15 horas semanais.

Até 1993, o estágio dos alunos era realizado unicamente em hospitais psiquiátricos. Em 1994, teve início o estágio em Ambulatório de Saúde Mental e a partir de 1995, também no CAPS. Anteriormente, a disciplina já convidava técnicos do CAPS para na EEUSP apresentar o serviço, falar do funcionamento da equipe e a inserção da enfermeira no modelo assistencial. Além disso, os alunos faziam uma visita ao serviço.

A partir do $2^{\circ}$ semestre de 1995 , quando já negociávamos o Convênio, passamos a ter o ensino sistematizado no CAPS. Nos anos de 1995 e 1996 passaram três grupos de alunos no serviço e houve em esforço da equipe técnica para absorvê-los nas atividades existentes. Apesar dos esforços, muitas foram as dificuldades. Havia um processo de negociação constante entre docentes, NEP e técnicos que ia desde o número de alunos que poderia freqüentar a "casa" até que atividades poderiam participar ou executar. Essa situação determinava quase uma reprodução do estágio hospitalar, onde a atividade do aluno é unicamente a interação um-aum com o paciente. Apesar disso, os alunos já avaliavam positivamente a experiência, principalmente pela proposta assistencial que o serviço tinha.

No ano de 1997, quando foram coletados os dados analisados, com o Convênio em andamento, tivemos um grupo de alunos no $1^{\circ}$ semestre. Encontramos problemas na aceitação de alunos de graduação nas atividades do Núcleo de Cuidados e Núcleo de Projetos, entretanto, o fato da docente participar ativamente da gestão do PIDA e do NEP favoreceu a superação das dificuldades, além disso, o fato de o CAPS ser formalmente, a partir do Convênio, uma instituição de ensino, quebrou resistências. O trabalho incansável do Grupo de Ensino permitiu uma recondução do processo de ensino para aquele grupo de alunos e também para o grupo do $2^{\circ}$ semestre.

$\mathrm{Na}$ avaliação formal do curso o grupo de alunos do $1^{\circ}$ semestre avaliou como aspecto positivo no modelo assistencial: a liberdade e autonomia dos pacientes: a diversidade de práticas para o aprendizado e o tratamento humanitário, no entanto, indicaram aspectos negativos relacionados aos "impedimentos" de aprendizado, como podemos ver nesta frase:

"Havia uma animosidade velada com relação à. nossa presença e participação nas atividades."
A partir desta avaliação, discutida no NEP e no Conselho Administrativo do PIDA, foram executadas algumas estratégias para o estágio do $2^{\circ}$ grupo, tais como: os alunos foram recebidos pela psicóloga, membro do Grupo de Ensino, para descrição das atividades que participariam no Núcleo de Cuidados e no de Projetos; foram esclarecidos acerca do funcionamento do serviço e o modelo de atenção. A mesma técnica voltou a reunirse com eles para confirmar os cronogramas, tirar dúvidas, entre outros. A enfermeira manteve um espaço de supervisão semanal e, de maneira geral, percebíamos, no decorrer do estágio um outro "clima", que pressupunha aceitação por parte de estagiários de graduação, aprimorandos, técnicos e docentes.

Frente a essa experiência, interessou-nos saber como o grupo de estagiários do segundo semestre vivenciou o PIDA, já mais estruturado, e como percebeu o modelo assistencial do serviço. Para isso, elaboramos um questionário (em anexo), que foi apresentado aos alunos, no último dia de estágio, quando ja haviam sido avaliados pela docente e feito a avaliação do estágio com a enfermeira e a psicóloga do serviço. Todos os alunos (07) quiseram participar do estudo e responderam individualmente o questionário, embora a participação fosse facultativa.

Todos afirmaram que o fato do ensino teóricoprático ocorrer num serviço que é campo de Projeto de Integração Docente Assistencial facilita o processo de aprendizado e que a participação do docente no serviço possibilita uma maior e mais fácil integração nas atividades e com os profissionais:

"Repercutiu positivamente, uma vez que a- docente sendo integrada ao serviço facilita a integração dos estagiários, no serviço e com, os profissionais"

"O professor estando interado com a instituição proporciona uma abertura maior para o aprendizado dos estagiários."

Da mesma forma que a inserção do docente no serviço através de um PIDA facilita o processo de ensino, o pressuposto da participação dos técnicos do serviço nesse processo também foi avaliado pelos alunos. De acordo com os alunos, a supervisão - um dos meios de participação da enfermeira do campo no ensino - contribuiu para o entendimento da filosofia do serviço além de ser esclarecedora das atividades desenvolvidas:

"Permitem uma visão mais ampla do serviço...” 
"A enfermeira do campo através das supervisões, contribuia através de relatos e mesmo conteúdos teóricos $e$ práticos para a nossa aprendizagem."

As afirmações dos alunos podem indicar que existe um grau de assunção e de mescla de papéis profissionais entre o agente da instituição formadora e o prestador de assistência e, também, fica claro que a "responsabilidade é compartilhada, porém sem significar igualdade de papéis assumidos" (EGRY; FONSECA, 1991).

A enfermeira teve uma participação mais ativa no processo, no entanto, os alunos estavam todo o tempo interagindo com outros trabalhadores e estagiários nas atividades assistenciais. De modo geral, afirmam que a interação com trabalhadores universitários, não universitários e outros estagiários foi positiva e houve possibilidades de trocas e de aprendizado dentro e fora dos grupos de trabalho. A interação com outros estagiários ocorreu de maneira tranqüila e proveitosa, assim como com trabalhadores de nível não universitário, pela possibilidade de trocas:

"No geral tive uma boa interação com. todos os funcionários. Estes se mostraram disponiveis para orientar, ajudar, e colaborar quando necessário. Sempre que precisei os trabalhadores me auxiliaram."

"Em relação aos trabalhadores não universitários foi legal porque pude perceber que eles desempenham de maneira brilhante suas atividades $e$ talvez com maior tranqüilidade."

Essas frases deixam claro que não existe uma simples utilização do serviço como campo de treinamento, com rígida divisão e manutenção dos papéis clássicos de docentes e assistenciais. A ocupação do espaço de IDA, nesta experiência de ensino, parece ter se dado "com múltiplos atores institucionais que, apesar da assunção de posições diferenciadas e específicas tanto no vínculo institucional do trabalho como na reação de subordinação dada pela hierarquia de cada instituição, buscam perfilar-se num caráter de igualdade diante da construção conjunta de uma nova qualidade de assistência e de ensino. Permeiam este processo não somente o saber acadêmico, como também o saber não acadêmico na condução do processo ensino-aprendizagem" (UNIVERSIDADE 1989/90).

Este PIDA privilegia a Reabilitação Psicossocial como concepção norteadora do campo de prática e de conhecimentos. Assim, procuramos saber dos alunos se, ao final do estágio, sabiam quais os conceitos que norteiam o modelo de atenção no CAPS.
Todos responderam que o conceito norteador é o da Reabilitação Psicossocial e completaram a resposta indicando os conceitos autonomia, poder de contratualidade, independência do usuário e inserção no trabalho e na sociedade. Um aluno ainda citou como conceito norteador do serviço o trabalho em equipe interdisciplinar. O PIDA Saúde Mental compreende Reabilitação Psicossocial como "um processo que determina o aumento da capacidade do usuário de utilizar oportunidades e recursos, um percurso que faça crescer sua contratualidade psicológica e social, construindo o direito pleno de cidadania e a constituição material de tal direito" (SARACENO,

1995).

O aprendizado dos alunos deu-se nos diversos campos de atividade do serviço que foram "se configurando e estruturando a partir da explicitação de necessidades diferenciadas dos usuários nos seus distintos percursos pela instituição" (GOLDBERG, 1995), entretanto, é no Núcleo de Projetos Especiais ou de Sociabilidade que a noção de Reabilitação fica mais explícita, pois "seus objetivos relacionam-se com projetos que buscam incluir o sujeito no convivio social mais amplo e expandir sua autonomia nos diversos niveis de relacionamento coletivo" (BARIÇÃO, 1994). E, ainda de acordo com GOLDBERG (1995), a "noção de reabilitação surgiu aos poucos no CAPS, como um aprendizado e uma conquista natural".

Os alunos participaram de pelo menos um projeto do Núcleo Terapêutico e um do Núcleo de Projetos ou de Sociabilidade, particularmente no Projeto Trabalho e parecem não descriminar claramente as diferenças conceituais entre eles. Acertadamente, identificam os projetos terapêuticos com momentos possiveis de expressão para o usuário: da fala, dos sentimentos, da "doença" a ser tratada. Enquanto nos projetos de Reabilitação Psicossocial do Núcleo de Sociabilidade, cuida-se da inserção social do paciente, de ampliar sua autonomia, também através de uma atividade produtiva:

"Creio que nos projetos terapêuticos há uma proposta de liberdade de expressão, do usuário que também, existe nos de Reabilitação Psicossocial mas que visa a responsabilidade do usuário".

"Nos projetos de Reabilitação Psicossocial o paciente aprende um determinado tipo de trabalho e nos projetos terapêuticos é discutido a psicopatologia do paciente"

Embora sejam capazes de relacionar corretamente os conceitos norteadores do modelo assistencial, o que denota grande habilidade associativa, ainda definem de forma simplista o 
projeto terapêutico institucional, aqui fortemente identificado com o senso comum. Com relação aos projetos de sociabilidade, vinculando-os apenas à noção de trabalho, denunciam uma representação social, dessa atividade em particular, forte e organicamente associada à praxisterapia (herdeira do Tratamento Moral), modelo diametralmente oposto ao desenvolvido no CAPS.

Ainda assim, afirmam que refletir sobre essas diferenças contribui para a formação, pois:

\section{"Mostrou que os dois tipos de projetos são indispensáveis no tratamento do paciente psiquiátrico.. " \\ "Contribuiu para ter uma visão mais ampla da assistência do paciente psiquiátrico e a importância desses aspectos na vida dele, além "de ter sido importante no desenvolvimento do estagio."}

Um dos objetivos do PIDA em Saúde Mental é a tentativa de superação do modelo tradicional de ensino e de assistência e oferecer resposta à necessidade de formação de profissionais com novas atitudes em relação ao processo saúde-doença mental. Para sabermos se essa experiência de ensino possibilitou alguma transformação, perguntamos aos alunos como percebiam o doente mental antes do estágio e como o descrevia ao final. De modo geral, o doente mental era visto de acordo com as representações do senso comum, ou seja, um indivíduo irrecuperável, agressivo, incapaz e irresponsável:

"Imaginava um. indivíduo agressivo $e$ violento. Tive medo e fiquei bastante angustiada por imaginar uma pessoa babando, gritando, etc."

"Como alguém, agressivo e sem capacidade para realizar qualquer tipo de atividade ou trabalho, já que eu acreditava que um doente mental não seria capaz de assumir responsabilidades."

Ao final do estágio contrapõem à esta representação - do indivíduo agressivo - um ser humano com limites e necessidades:

"Como ser humano como outro qualquer, com suas limitações, carências, enfim uma pessoa que deva ter todos os direitos e oportunidades para ter uma vida digna.."

"Como uma pessoa que precisa. de ajuda terapêutica e de suporte social para que possa desenvolver seu poder de contratualidade."
Do indivíduo agressivo para um sujeito doente com potencialidades. A mudança ocorrida na representação do objeto/sujeito de cuidados é acompanhada de instrumentos/meios de intervenção para este cuidar. Os alunos, em sua maioria, relataram que, antes do estágio, pensavam como tratamento mais importante o farmacológico:

"Um tratamento baseado, principalmente e quase que unicamente emir. psicofármacos."

"Medicamentos e psicoterapias. "

Ao término do estágio, outros meios são indicados:

"Além dos medicamentos e das psicoterapias, considero importante a questão da reabilitação psicossocial desse individuo, a questão da autonomia, dos direitos e deveres""

"Reinserção social, apoio familiar,
acompanhamento de uma equipe
multiprofissional e relacionamento
interpessoal e efetivo."

Uma aluna reflete que diferentemente da clínica geral, onde as intervenções são feitas rapidamente e com resultados mais imediatos obedecendo a lógica da prática clínica hegemônica, pautada no princípio positivo da causalidade e respostas universalizadas para universos nosográficos definidos e inflexíveis, no acompanhamento dos sujeitos acometidos por transtornos mentais de qualquer ordem, as intervenções obedecem uma lógica de outra ordem, onde o processo saúde-doença é intransferivel e singular, onde não é possivel uniformizar sintomas e afetos, onde cada sujeito é um universo parcial do universo dos humanos. Chega, então, a uma sintese dos pressuposotos do modelo assistencial do CAPS:

"Vejo que é um trabalho mais demorado $e$ subjetivo, que requer paciência $e$ empatia."

E, complementando a deixa da aluna, afirmamos que esse trabalho requer também investimento teórico-conceitual, disponibilidade interna e externa dos operadores em aprender com a dor do outro a sua prápria dor.

\section{CONSIDERAÇÕES FINAIS}

PITTA (1996) afirma que "nos tempos atuais, avaliar experiências tem se constituído um campo fértil e criativo de exercício da imaginação de pesquisadores e gerentes de experiências ou 
projetos" e, assim, essa foi a primeira experiência na tentativa de avaliação do ensino de graduação em enfermagem psiquiátrica da EEUSP num PIDA em Saúde Mental.

A análise obtida indica que o IDA tem a preocupação de buscar a convergência entre a instituição formadora e a prestadora de assistência à saúde mental. Podemos afirmar que aconteceram mudanças no processo de aprendizado dos alunos do primeiro semestre letivo de 1997, em relação ao do anterior e acreditamos que as transformações foram determinadas pelo modelo assistencial no CAPS.

Neste modelo a preocupação maior é a construção de um projeto terapêutico compartilhado e participativo, que não pressupõe a perda da identidade profissional mas a relativação da prática específica no coletivo (SILVA; FONSECA, 1995). Essa nova reação, segundo as autoras, redimensiona a relação dos prestadores de assistência entre si e com o usuário, possibilitando a construção de uma parceria entre o saber científico (do profissional) e o saber aico (do usuário individual e familiar).

Uma conseqüência, apontada pelos alunos, diz respeito a uma maior implicação dos parceiros (profissional e usuário individual e coletivo) na construção do projeto terapêutico individualizado, ou seja, um projeto de longo prazo, descortinado com o tempo e com a confiança, adquiríveis somente na vigência de uma relação e vínculo afetivo comprometido com a transformação das subjetividades envolvidas. Acredita-se que somente o encontro amoroso com o outro viabiliza a transformação do ser humano e tratar, cuidar, a mparar, sustentar, assegurar, são fundamentalmente ações amorosas.

Podemos afirmar que essa experiência redimensionou a relação dos alunos com usuários, técnicos e sua identidade profissional: pelas trocas efetivas, pela diversidade de intervenções, pelo aprendizado e interlocução com o serviço, pela inserção do docente na gestão do projeto, transformando o processo ensino-aprendizagem num encontro criativo e reflexivo.

\section{REFERENCIAS BIBLIOGRÁFICAS}

BARIÇÃO, S. M. F. Centro de Atenção Psicossocial: construindo um projeto. Saúde Atual, v.1, n.1, 1994.

BARROS, S. O louco, a loucura e a alicnação institucional: o ensino de enfermagem sub judice. São Paulo, 1996. 201p. Tese (Doutorado) - Escola de Enfermagem da Universidade de São Paulo.

CAVALCANTI, M. T. Transformações na assistência psiquiátrica ou uma assistência psiquiátrica em transformação. In: SILVA FILHO, J. F.; RUSSO, J. (org.) Duzentos anos dc psiquiatria. Rio de Janeiro, Relume Dumaré. 1993, p. $145-60$.

EGRY, E. Y.; FONSECA, R. M. G. S. da. Integração DocenteAssistencial como estratégia de intervenção no contexto do ensino e da prática das profissões de saúde. In: ENCONTRO SOBRE O ENSINO PARA A CONSTRUCC̃̃ DE NOVOS REFERENCIAIS DO PROCESSO PEDAGÓGICO. São Paulo, 1991. Anais, São Paulo, Escola de Enfermagem da USP, 1991.

PITTA, A. M. F. Cuidados com o cuidar em saúde mental: estratégias. de avaliação. Cad. IPUB n.3 (suplemento) 1996. $12 \mathrm{p}$.

PITTA, A. M. F. et al. Projeto de Integração Docente Assistencial em Reabilitação Psicossocial. São Paulo, 1994 (mimeografado)

REZENDE, A. L. M. Saúde: dialética do pensar e do fazer. São Paulo, Cortez, 1986.

SARACENO, B. Reabilitação Psicossocial: uma estratégia para a passagem do milênio. In: PITTA, A.M.F.(org) Reabilitação Psicossocial no Brasil. São Paulo, Hucitec, 1996. P.33-47.

SILVA, A. L. A. e O Projcto Copiadora do CAPS: do trabalho de produzir coisas à produção de vida. São Paulo, 1997. 161p. Dissertação (Mestrado) - Escola de Enfermagem da Universidade de São Paulo.

SILVA, A. L. A. e ; FONSECA, R. M. G. S. da A questão da interdisciplinaridade. Rev. Bras. Enf, v. 48, n. 3, p. 212-17, 1995.

UNIVERSIDADE DE SÃO PAULO. Escola de Enfermagem. Departamento de Enfermagem em Saúde Coletiva. Integração Docente - Assistencial como referencial teórico-metodológico para o ensino e a pesquisa da assistência de enfermagem em saúde coletiva. (relatório preliminar de pesquisa). São Paulo, $1989 / 1990$

UNIVERSIDADE DE SÃO PAULO. Escola de Enfermagem: Projeto de Integração Docente Assistencial EEUSP e Município de Taboão da Serra: documento de proposta conjunta de trabalho. São Paulo, 1988 (mimeografado). 
ANEXO

\title{
PROGRAMA DE INTEGRAÇÃODOCENTE-ASSISTENCIAL - USP/SES Centro de Atenção Psicossocial Prof. Luiz da Rocha Cerqueira
}

\author{
Avaliação do ensino teórico-prático de Enfermagem Psiquiátrica
}

1. $\quad 0$ fato do CAPS ser um PIDA repercutiu no desenvolvimento do estágio? Explique.

2. Na sua opinião a enfermeira do campo contribuiu para o seu processo de aprendizado no estágio? Justifique.

3 Como foi a sua interação com os outros trabalhadores no CAPS?

- estagiários deoutros cursos;

- trabalhadores de nível universitário;

- trabalhadores não universitários.

4. Quais os conceitos que norteiam o modelo de atenção do CAPS?

5. Foi possivel perceber diferenças entre os Projetos Terapêuticos e os de Reabilitação Psicossocial? Quais?

6. No que o conhecimento dessas diferenças contribuiu para a sua formação?

7. Como você descreveria o doente mental antes de realizar o estágio?

8. Ao término do estágio, como você descreve o doente mental?

9. Antes do estágio, que aspectos você consideraria mais importantes no tratamento do doente mental?

10. Ao término do estágio, quais as pectos você considera mais importantes no tratamento do doente mental?

14. Que sugestões você tem para o ensino e para o campo, com o objetivo de facilitar o processo ensino-aprendizado? 